\title{
Tumor-Stroma Interactions in Bone Metastasis: Molecular Mechanisms and Therapeutic Implications
}

\author{
HanqIU Zheng, ${ }^{1,2}$ WenYang Li, ${ }^{1}$ and Yibin Kang ${ }^{1}$ \\ ${ }^{1}$ Department of Molecular Biology, Princeton University, Princeton, New Jersey 08544 \\ Correspondence: ykang@princeton.edu
}

\begin{abstract}
Metastasis and associated complications are the major cause of death for cancer patients. The incidence of bone metastasis is among the highest in cancers arising from breast, prostate, and lung. Common skeletal-related events caused by bone metastasis include aberrant bone remodeling (osteolytic, osteoblastic, and mixed), bone pain, fracture, spinal cord compression, and life-threatening hypercalcemia. It is now known that interactions between tumor cells and bone stroma lie at the core of major steps of bone-metastasis progression. Approved pharmaceutical drugs for the treatment of bone metastasis, including bisphosphonate and denosumab, were designed to target bone stromal cell components. In recent years, research in our laboratory and others has revealed intricate tumor-stroma interactions as the driving force behind osteolytic bone-metastasis development, providing a set of new candidates for future drug development. Moreover, recent studies also indicate existence of distinct bone niches in supporting hematopoietic stem cell renewal and differentiation. These niche components are likely utilized by metastatic cancer cells for seeding, progression, and therapy resistance of bone metastasis. Future studies in this direction may discover additional therapeutic targets for bone-metastasis treatment.
\end{abstract}

Metastasis is responsible for most of cancer-related morbidity and mortality (Wan et al. 2013). Bone is one of the prominent organs that suffered from the metastatic spread of cancer. In breast and prostate cancers, it is estimated that $>70 \%$ of late-stage patients develop skeletal metastasis (Mundy 2002; Weilbaecher et al. 2011). Bone is also extensively involved in many malignancies originating from hematopoietic cells, such as multiple myeloma and lymphomas (Melton et al. 2005; $\mathrm{Wu}$ et al. 2014). The common skeletal-related events (SREs) for bone metastasis include aberrant bone remodeling (osteolytic, osteoblastic, and mixed bone metastasis), bone pain, fracture, spinal cord compression, and life-threatening hypercalcemia (Mundy 2002; Weilbaecher et al. 2011; Ren et al. 2015). The initial clinical presentation and diagnosis for bone metastasis, including increased calcium and alkaline phosphatase levels and pain, could be varying for patients. The diagnosis and confirmation for bone metastasis involve regular X-ray imaging, bone scintillation, biopsy, and advanced medical imaging such as computerized tomography (CT), magnetic resonance imaging (MRI), and positron emission tomography (PET).

Once bone metastasis is confirmed, the goals of therapeutic treatments and management are to alleviate bone compression and bone pain, maintain intact bone structure and function, and slow down the metastatic tumor progression, although it is unlikely that the tumor cells can be completely eliminated from the bone. To achieve these goals, a set of treatment options can be personalized to meet the specific needs of each patient. For example, pain-relieving medicine/analgesia is commonly administrated to patients along with other systematic or localized treatments, including osteoclast inhibitors (bisphosphonate and denosumab), hormone therapy, chemotherapy, localized radiation, bone-targeting radioactive pharmaceuticals, and surgeries for patients with high risks of developing fractures. Most of these options are generic treatments for killing tumor cells or maintaining the quality of life for patients. Only bisphosphonate and denosumab are specifically designed for treating bone metastasis based on the molecular mechanisms of bonemetastasis development - by blocking osteolytic activity of the bone.

The exact molecular mechanisms underlying bone-metastasis progression could be varying in different cancer types. However, we now know that tumor-stroma interaction in the bone is critical for bone metastasis. In normal bone homeostasis, bone-building osteoblasts and bone-degrading osteoclasts keep their delicate balance to maintain the normal healthy bone structure. This balance is in part controlled by the secretion of an appropriate level of RANKL (receptor activator of nuclear factor $\kappa \mathrm{B}$ ligand) by osteoblast, which signals to RANK receptor in preosteoclasts to promote their maturation (Fig. 1). However, when bone metastasis develops, tumor cells often break the balance between the two bone cell types by signaling interaction with osteoblasts and osteoclasts. For example, in osteolytic bone metastasis of breast cancer, tumor cells can increase RANKL level in the microenvironment either by directly secreting RANKL or by inducing RANKL production by osteoblasts. Bone

\footnotetext{
${ }^{2}$ Present address: Department of Basic Medical Sciences, School of Medicine, Tsinghua University, Beijing, China

(C) 2016 Zheng et al. This article is distributed under the terms of the Creative Commons Attribution-NonCommercial License, which permits reuse and redistribution, except for commercial purposes, provided that the original author and source are credited.
} 


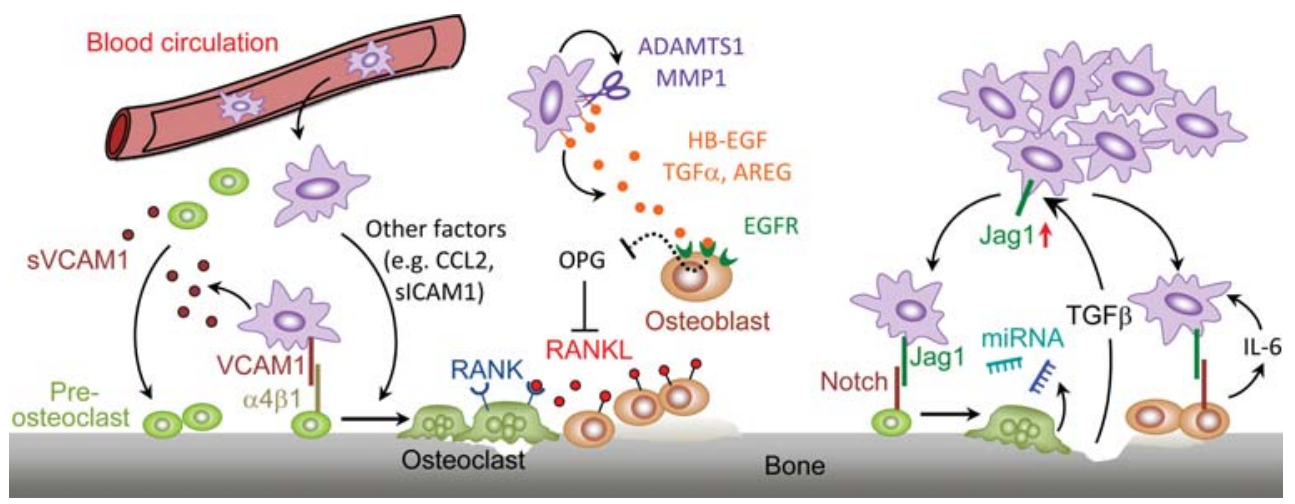

Figure 1. Tumor-stroma interactions in bone metastasis. Key pathways uncovered by our laboratory are highlighted here, including (1) VCAM1-activated osteolytic expansion of indolent bone micrometastasis (left); (2) osteolytic paracrine signaling cascade initiated by matrix metalloproteinases (MMPs, middle); (3) a positive-feedback loop in bone metastasis mediated by Jagged1/Notch and transforming growth factor $\beta$ (TGF- $\beta$ ) signaling pathways (right). See text for details. sVCAM1, soluble vascular cell adhesion molecule 1; CCL2, chemokine (C-C motif) ligand 2; sICAM1, soluble intercellular adhesion molecule 1; IL-11, interleukin 1; CTGF, connective tissue growth factor; ADAMTS1, ADAM metallopeptidase with thrombospondin type 1 motif 1 ; HB-EGF, heparinbinding epidermal growth factor-like growth factor; AREG, amphiregulin; EGFR, epidermal growth factor receptor; OPG, osteoprotegerin; RANK, receptor activator of nuclear factor $\kappa \mathrm{B}$; RANKL, receptor activator of nuclear factor $\kappa \mathrm{B}$ ligand; Jag1, Jagged1; miRNA, microRNA; IL-6, interleukin 6. (Reprinted, with permission, from Kang 2016.)

resorption by osteoclasts also leads to the release of many bone-derived growth factors and cytokines that feed back to tumor cells to promote tumor cell survival and growth in the bone. This extremely simplified model exemplified a powerful feed-forward loop of bone degradation and tumor growth process termed the "vicious cycle of bone metastasis" (Mundy 2002; Weilbaecher et al. 2011; Ell and Kang 2012). Bisphosphonate, by binding to bone surface and inhibiting osteoclast activity, protects bone from degradation. Similarly, denosumab, a RANKL-neutralizing antibody, blocks the binding of RANKL to its receptor on preosteoclasts, thus reducing the number of mature osteoclasts and inhibiting bone resorption (Baron et al. 2011).

Recent studies in the field, including work done in our laboratory, revealed multiple layers of tumor-stroma interactions in bone metastasis of breast cancer. Our study revealed multiple molecular and signaling pathways promoting bone-metastasis progression, including matrix metalloproteinase 1 (MMP1) and a disintregin and metalloproteinase with thrombospondin motifs 1 (ADAMTS1), vascular cell adhesion molecule 1 (VCAM1), transforming growth factor $\beta$ (TGF- $\beta$ ) pathway and Jagged1NOTCH pathway, intercellular adhesion molecule 1 (ICAM1), and microRNAs (Fig. 1). These results provide important candidate molecules as targets for treating bone metastasis.

\section{METASTASIS TO BONE IS DRIVEN BY A UNIQUE REPERTOIRE OF BONE-METASTASIS GENES}

The development and widespread application of the genomic profiling method at the turn of the century opened up the possibility of search for organotropic metastasis genes in a comprehensive and unbiased way.
However, there was a very limited number of breast cancer cell lines capable of generating bone metastasis reliably in mouse models. The Massagué group applied the in vivo selection strategy to derive highly bone-metastatic variants from the heterogeneous MDA-MB-231 human breast cancer cell line that was established from the pleural effusion of a metastatic breast cancer patient (Fig. 2A; Cailleau et al. 1978). Tumor cells were injected into the left ventricle of nude mice to generate bone metastasis. When bone metastasis could be detected by $\mathrm{X}$-ray imaging, tumor cells were isolated from bone lesions for in vitro expansion (Kang et al. 2003). The process can be reiterated for further enrichment of bone-metastatic variants and to quantify the metastatic efficiency of each subline. Using the parental MDAMB-231 and 12 subpopulations with differential bonemetastatic potentials, a gene signature was generated that was linked to bone-metastatic potential, including 43 up-regulated genes and 59 down-regulated genes. Interestingly, the majority of the mostly highly enriched genes encode membrane or secretory proteins, including chemokine receptor CXCR4 for bone-homing, angiogenic factor fibroblast growth factor-5 (FGF5), osteoclast stimulating factor interleukin-11 (IL-11), and osteopontin (OPN), as well as MMP1 and ADAMTS1 (Fig. 2B). This specific pattern of gene enrichment associated with bonemetastatic ability indicates that tumor-stroma interactions mediated by secreted factors are likely to be critical for tumor cells to survive and thrive in the bone microenvironment. A few of these candidate molecules have indeed been functionally validated to be important for bone metastasis.

An interesting question is how do these sublines with various bone-metastatic abilities emerge from in vivo selection? Do they preexist in the original cancer cell line because of tumor heterogeneity, or do they evolve and acquire the bone tropism de novo during in vivo 
A
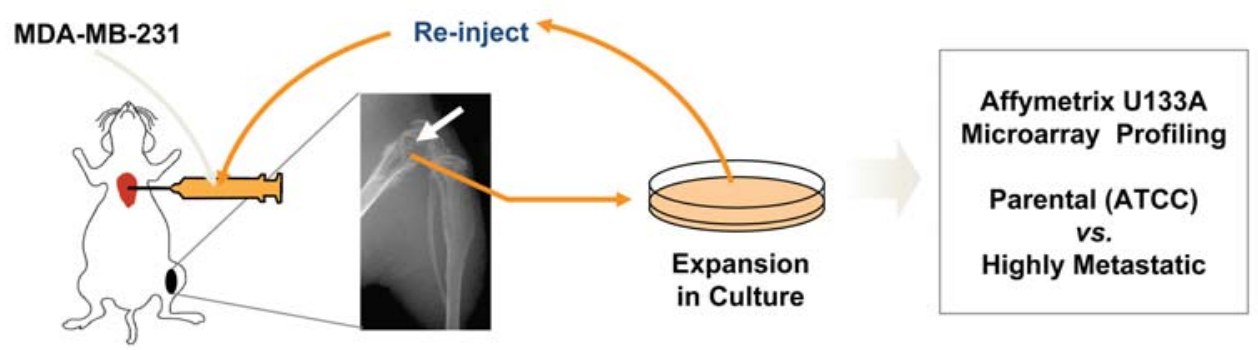

B
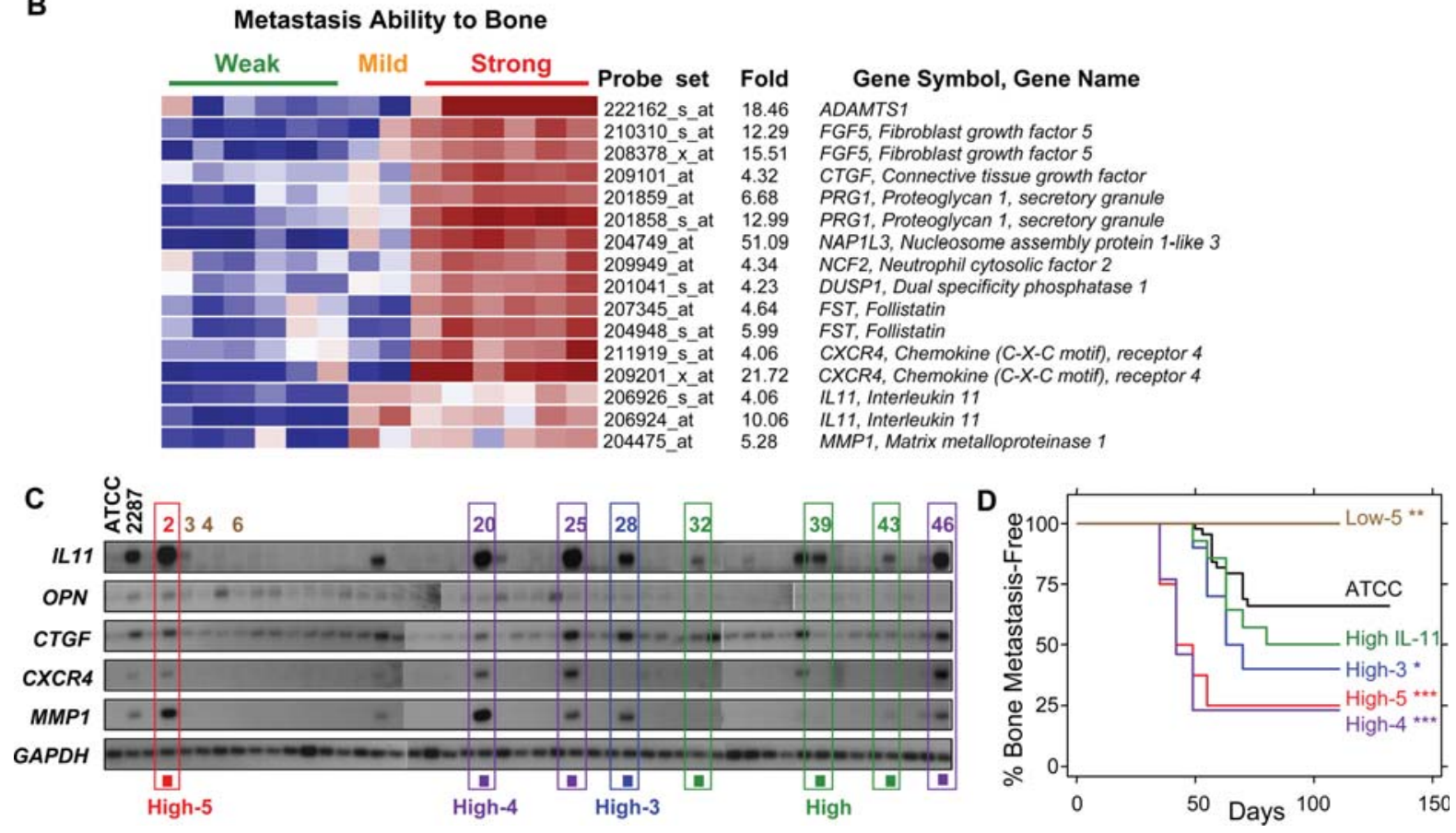

Figure 2. Discover bone-metastatic genes. (A) Procedures of in vivo selection for bone-metastatic cells. Parental MDA-MB-231 cells were inoculated into the left cardiac ventricle of nude mice. Tumor cells were isolated and expanded from X-ray imaging-confirmed bone lesions and reselected for another round. Reisolated cells were expanded and injected into nude mice to gauge their metastatic potentials. $(B)$ RNAs from these generated cell lines were subjected to gene expression profiling analysis to identify candidate metastasis-enhancing genes. A subset of hierarchical cluster of 11 genes (with 16 probes) from the original 127 probe sets, which differed by more than fourfold in expression level between weak and strong metastatic groups. $(C)$ Randomly picked single clones from the parental MDA-MB-231 population were expanded in culture, and subjected to northern blot analysis for five bone-metastasis genes. (D) Kaplan-Meier plot displays the bone-metastatic incidence of the parental MDA-MB-231 population and the indicated single-cell progeny clones. ${ }^{*}, P<0.05$; ${ }^{* *}, P<0.001$, compared with parental cells. (Adapted from Kang et al. 2003, with permission from Elsevier.)

selection in mice? The preexisting model seems to be more realistic as the selection time in the mouse is relatively short $(6-10 \mathrm{wk})$. To confirm this, 46 single-cellderived clonal populations (termed SCP for "single-cell progenies") were isolated in vitro from parental MDAMB-231 cells and the expression level of five major bonemetastasis signature genes were measured. Although most SCPs do not express any of the bone-metastasis genes, a few SCPs express various numbers of the five genes at high levels (Fig. 2C). Remarkably, the bonemetastatic potential of these SCPs correlated well with their different degree of metastasis gene expression (Fig. 2D). This result not only confirmed that high-metastatic variants preexist in the heterogeneous population of the parental MDA-MB-231 cell line but also underscored the cooperative nature of metastasis genes in promoting the metastatic behavior of cancer cells.

\section{ADAMTS1 AND MMP1 SYNERGISTICALLY PROMOTE METASTASIS BY ALTERING CYTOKINE PRODUCTION IN THE MICROENVIRONMENT}

With the identification of these secreted proteins and cell surface proteins in highly bone-metastatic cell lines, we started to systematically evaluate the functional importance of these individual genes in the bone metastasis signature and explore the therapeutic options for targeting these genes. We first focused our analysis on two metal- 
loproteinases, MMP1 and ADAMTS1 (Lu et al. 2009), which are among the two highest expression genes in the bone-metastasis gene signature (Fig. 2B). The expression of MMP1 and ADAMTS1 were knocked down separately or in combination by short-hairpin RNAs (shRNAs) in SCP20 cells, a highly bone-metastatic variant of MDAMB-231 (Fig. 2C,D). These cells were then intracardially (IC) injected into nude mice to generate bone metastasis. Interestingly, single knockdown of either MMP1 or ADAMTS1 did not show any bone-metastasis reduction. However, combined knockdown of both genes led to a drastic reduction in bone-metastasis progression (Fig. $3 \mathrm{~A}$ ), as showed by in vivo bioluminescence imaging (BLI) (Fig. 3B). X-ray imaging and tartrate-resistant acid phosphatase (TRAP) staining for osteoclast cells revealed that reduced bone metastasis is accompanied by smaller osteolytic area and decreased TRAP-positive osteoclasts (Fig. 3B). Using in vitro assays for osteoclast differentiation from primary bone marrow cells, we concluded that MMP1 and ADAMTS1 altered the production of secreted growth factors from tumor cells that signal to osteoblasts to reduce the expression of osteoprotegerin
A

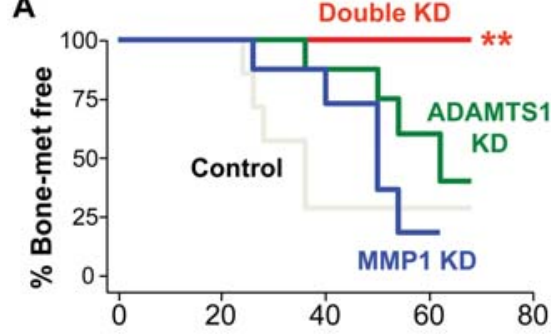

C

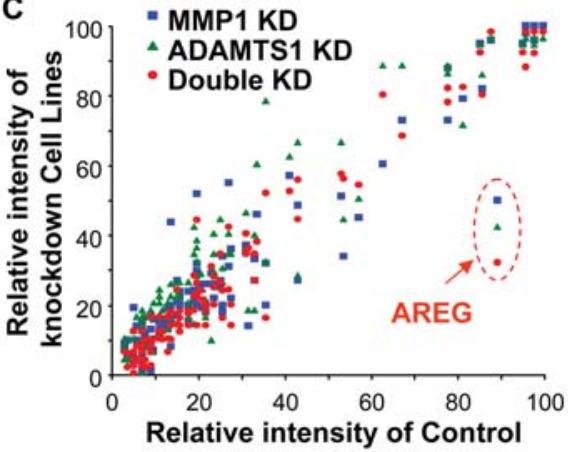

B

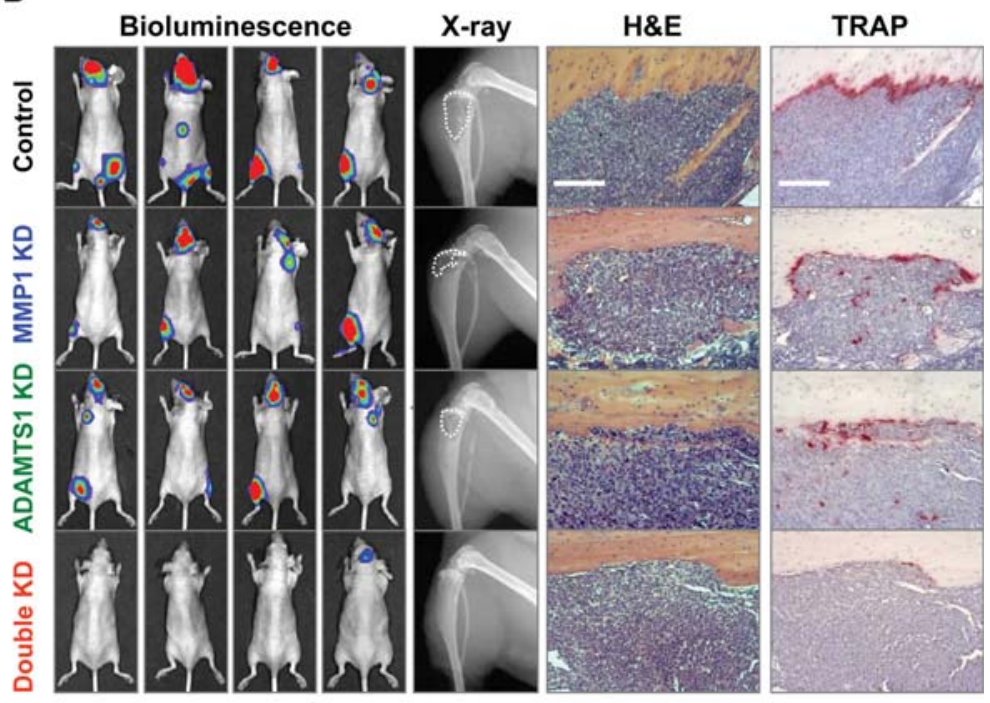

D

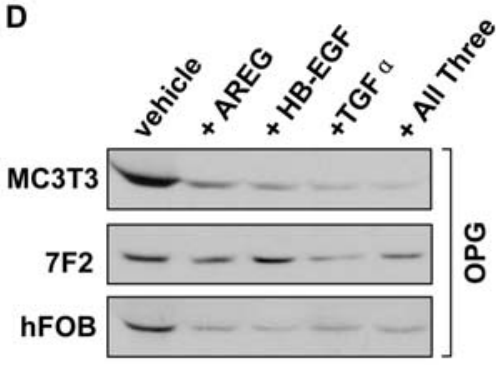

E

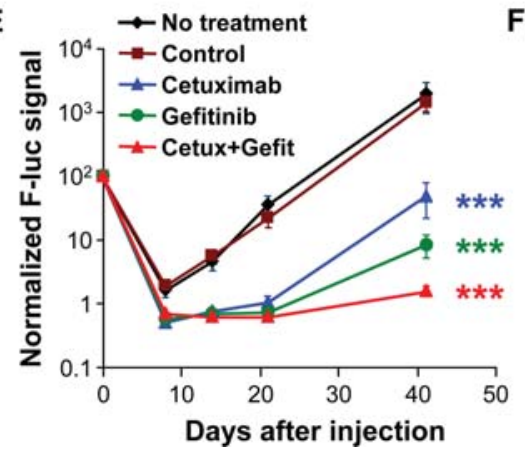

$\mathbf{F}$

\begin{tabular}{lc} 
Treatment & Bone met \\
\hline Control & $10 / 10$ \\
Cetuximab & $3 / 10$ \\
Gefitinib & $3 / 10$ \\
Cetux+Gefit & $0 / 10$ \\
\hline
\end{tabular}

Figure 3. Combined knockdown (KD) of ADAMTS1 and MMP1 inhibits bone metastasis. $(A)$ Kaplan-Meier plot indicates the incidence of bone metastases after intracardiac injection of various cell lines in immune compromised nude mice, including SCP20 stably transduced with control short-hairpin RNA (shRNA) or shRNAs targeting MMP1, ADAMTS1, or both. $n=10 .{ }^{* *}, P<0.01$, based on log-rank test. $(B)$ Representative bioluminescence, radiographic, and histological (H\&E and TRAP staining) images from each group at day 50 postinjection. $(C)$ A human cytokine array kit was used to determine the differentially expressed cytokines in the conditional media from either MMP1 or/and ADAMTS1 knockdown or control SCP20 cells. AREG was the most down-regulated protein in knockdown cells. (D) Treatment of recombinant AREG, HB-EGF, and TGF- $\alpha$ individually or in combination in three different osteoblast cells repressed OPG expression as measured by immunoblotting. (E) In the SCP20 bone-metastasis model, single or combined treatment of cetuximab and gefitinib decreased bone-metastasis progression as measured by bioluminescence. $(F)$ Single or combined treatment of cetuximab and gefitinib decreased the incidence of bone metastasis in nude mice. ADAMST1, a disintegrin and metalloproteinase with thrombospondin motifs 1; MMP1, matrix metalloproteinase 1; H\&E, hematoxylin and eosin; TRAP, tartrate-resistant acid phosphatase; HB-EGF, heparin-binding epidermal growth factor; TGF- $\alpha$, transforming growth factor $\alpha$; hFOB, human fetal osteoblastic; OPG, osteoprotegerin. (Adapted, with permission, from Lu et al. 2009, Cold Spring Harbor Laboratory Press.) 
(OPG), a natural decoy receptor and inhibitor of RANKL. Through cytokine array profiling, we observed a significant reduction of Amphiregulin (AREG) in the conditional media from MMP1 and/or ADAMTS1 knockdown cells, with the most profound reduction in double knockdown cells (Fig. 3C). AREG belongs to the epidermal growth factor (EGF) family ligands, and its membrane form can be proteolytically released by MMP1 and ADAMTS1 into the microenvironment for paracrine signaling. Recombinant AREG and its related family members were able to reduce OPG expression in osteoblasts (Fig. 3D) and subsequently boost osteoclast differentiation. These results thus underscored the potential application of EGF receptor (EGFR) inhibitors in bone-metastasis treatment. To this end, we performed preclinical testing of EGFR inhibitors, cetuximab and gefitinib, in bone-metastasis models. Indeed, treatment with either cetuximab or gefitinib or both in combination significantly reduced bone metastasis incidence and metastatic tumor burden. (Fig. 3E,F). These studies presented proof of concept that inhibiting EGFR signaling in tumor-stroma cross talk using a clinically approved EGFR inhibitor may reduce bone-metastasis progression.

\section{TARGETING TGF- $\beta$ AND Jagged1-Notch SIGNALING PATHWAYS IN BONE METASTASIS}

TGF- $\beta$ is one of the most abundant growth factors stored in the bone and has been suspected to be released during bone destruction to provide a feedback signal to cancer cells to promote their metastatic behavior. To directly determine whether TGF- $\beta$ signaling is activated during bone metastasis, we developed a xenograft system in which we could detect TGF- $\beta$-SMAD pathway activation status while simultaneously monitoring tumor burden in the bone. In this system, tumor cells were transduced with a constitutive Renilla luciferase (RLuc) expression cassette to monitor tumor burden and served as an internal control for normalizing tumor burden, whereas a TGF- $\beta$-responsive element was placed upstream of the firefly luciferase (F-Luc) coding sequence to provide a direct readout of TGF- $\beta$ signaling intensity in cancer cells (Fig. 4A; Korpal et al. 2009). When the tumor cells were injected to nude mice via the left cardiac ventricle to form bone metastasis, the relative level of F-Luc/R-Luc gradually increased over the course of bone-metastasis progression with the development of osteolytic lesions, whereas no such increase was observed in nonosteolytic lesions (Fig. 4B,C). Furthermore, the increase of F-Luc activity could be blocked by treating the mice with bisphosphonate, which inhibits osteoclastogenesis and prevents osteolytic degradation of the bone. The result suggests that TGF- $\beta$ pathway activation in tumor cells is enhanced by TGF- $\beta$ released from osteolytic bone degradation. Blocking this feedback loop using TGF- $\beta$ receptor kinase inhibitors thus could potentially be used to slow down bone-metastasis develop- ment. Interestingly, although early treatment of mice with TGF- $\beta$ inhibitors can significantly reduce bone-metastasis burden, late treatment of established lesions can reduce TGF- $\beta$ signaling activity but did not result in a reduction of tumor burden. These findings highlight the notion that the therapeutic window is critical to achieve the optimal chemotherapeutic outcome of bone-metastasis-targeting agents.

We noticed the Notch ligand, Jagged1, is among the genes that is regulated by the TGF- $\beta$-Smad pathway and was also highly expressed in bone-metastatic breast cancer cell lines (Fig. 4D,E; Sethi et al. 2011). Furthermore, elevated expression of Jagged1 in primary breast tumors is associated with higher risk of bone metastasis in patients. The Notch pathway has been extensively studied for its roles in bone development (Engin et al. 2008; Hilton et al. 2008) and developmentally conserved pathways are well-known to be often hijacked by cancer cells to promote their malignant behaviors (Sethi and Kang 2011). Therefore, we explored whether the Jagged1Notch pathway is functionally involved in breast cancer bone metastasis. shRNA-mediated stable knockdown of Jagged 1 expression in highly metastatic breast cancer cell lines drastically reduced bone-metastasis burden (Fig. $4 \mathrm{~F}, \mathrm{G})$. On the other hand, overexpression of Jagged1 in weakly bone-metastatic cells promoted bone-metastasis progression. A close examination of the bone tissues showed that Jagged1-overexpressing cells generated much larger bone lytic areas with increased mature osteoclast numbers in the bone (Fig. 4F,G).

To understand the molecular mechanism by which Jagged1 promotes bone metastasis, we decided to coculture tumor cells with either osteoblasts or preosteolcasts in vitro. We discovered that tumor-derived Jagged1 interacts with osteoblast cells to induce Notch activation and IL-6 production, which in turn feeds back to tumor cells to promote their proliferation (Fig. 4H,I). This feedback loop could be completely blocked by either knockdown of Hey1, a Notch downstream effector gene in osteoblasts, or by administration of $\gamma$-secretase inhibitor (GSI, MRK-003 from Merck) to block Notch activation in the coculture system (Fig. 4H). Jagged1 also promotes osteolytic bone metastasis by engaging Notch signaling in osteoclasts. Tumor-derived Jagged1 induces the osteoclastogenesis through which monocytic preosteoclasts fused to form large multinucleated, TRAP-positive osteoclasts within several days in cell culture. This osteoclast maturation process could be completely diminished by administration of the GSI (Fig. 4J). In vivo administration of GSI inhibitor also dramatically decreased bone-metastasis burden, osteolytic lesion area, and osteoclast cell number in the bone (Fig. 4K). Although it is now known that GSIs have severe adverse effects on the gastrointestinal track of the patients, future modification of the inhibitor could potentially alleviate some of these side effects. Moreover, the development of pharmaceutical agents specifically targeting Jagged 1 while sparing other Notch ligand may achieve similar therapeutic benefit without the adverse side effects incurred by pan-Notch inhibition by GSI. 
A

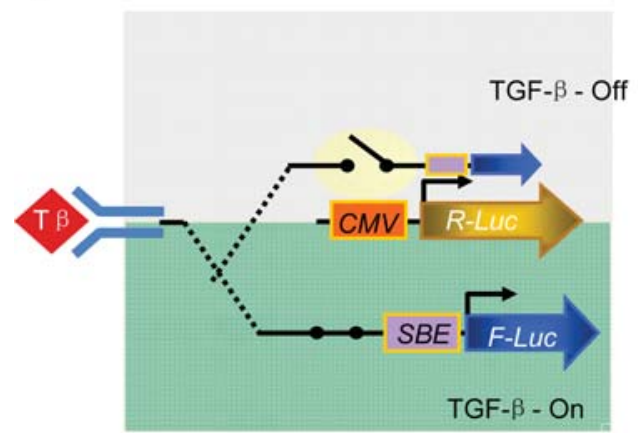

B

Rtuc (Tumor Burden) (TGFb Activity)
C

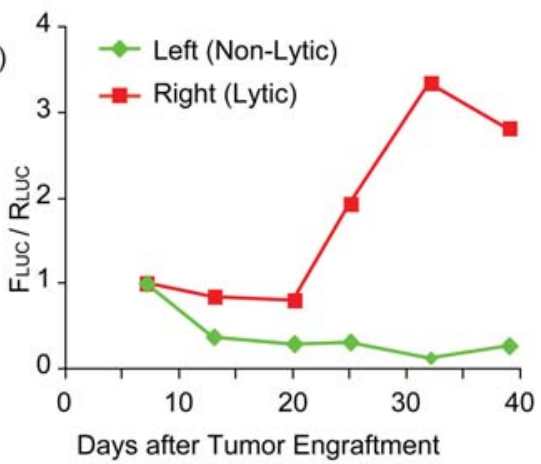

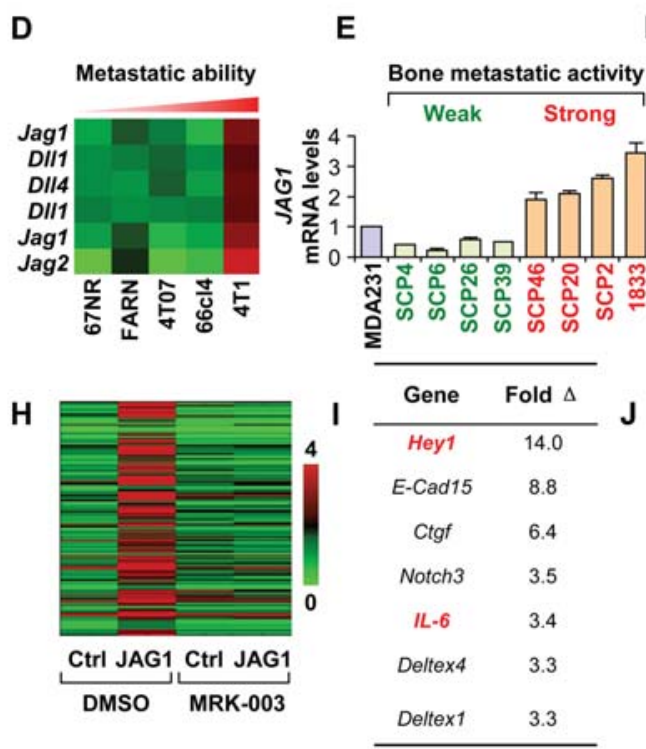
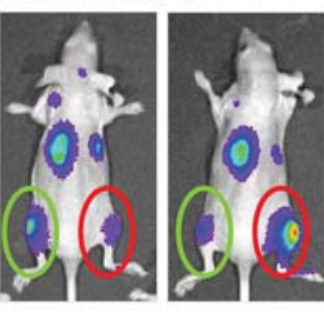

F
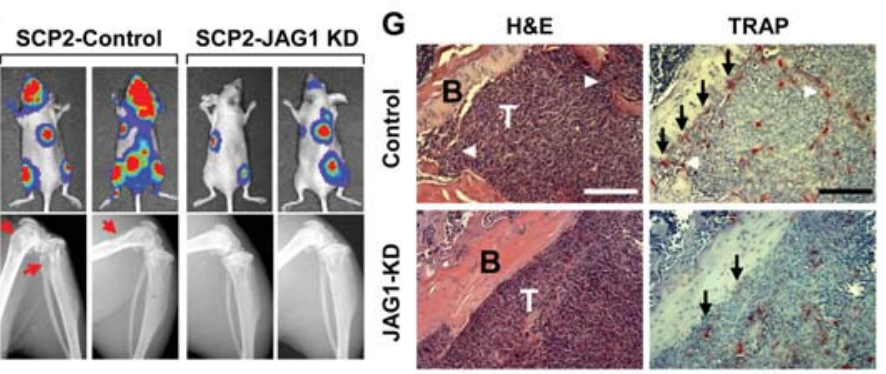
J

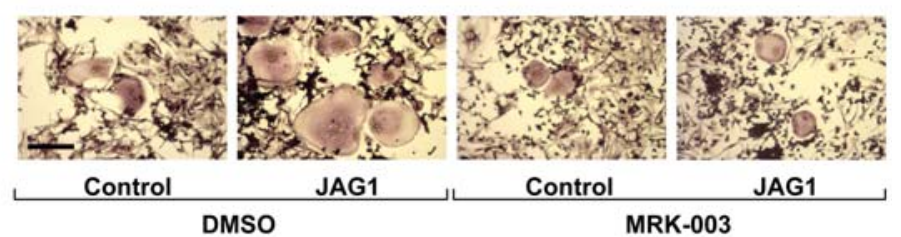

K
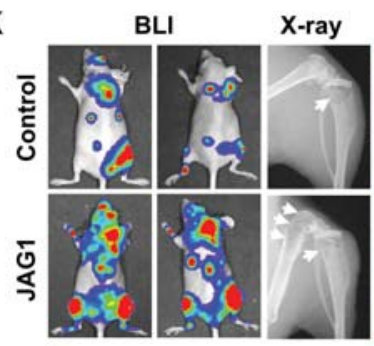

H\&E

TRAP

BLI

X-ray

H\&E

TRAP

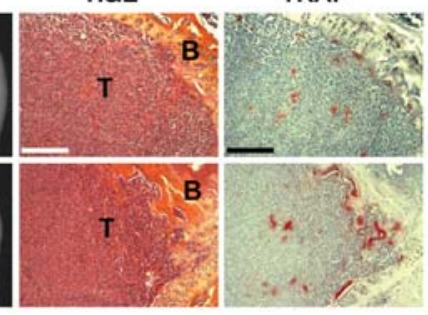

Vehicle
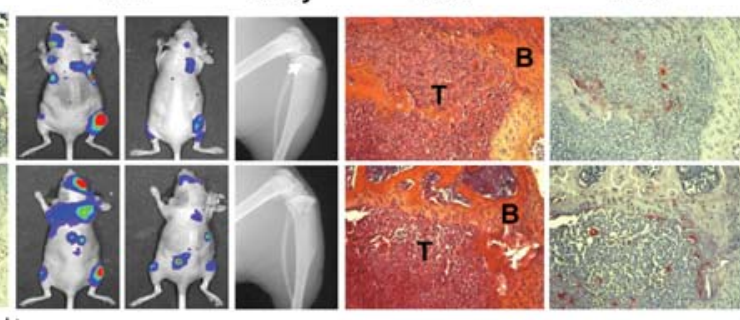

MRK-003

Figure 4. TGF- $\beta$ and Jagged 1 -Notch pathway in bone-metastasis progression. $(A)$ A schematic representation of the dual-luciferase reporter system to track tumor burden and TGF- $\beta$ pathway activation and to conditional control of TGF- $\beta$-Smad signaling activity. $(B)$ Osteolytic bone metastasis (right leg) displayed enhanced TGF- $\beta$ pathway activation compared with the nonosteolytic lesion (left leg). (C) Quantification of normalized F-Luc/R-Luc intensity from mouse in $B$. (D) Heatmap of gene-expression profiling of Notch ligands in the 4T1 series with differential bone-metastatic potentials. $(E)$ The mRNA levels of Jagged 1 in parental MDA231 cell and its derivative sub-cell lines with differential bone-metastatic potentials. $(F)$ Control or Jagged1 knockdown SCP2 cells were intracardially injected into nude mice for bone metastasis. Metastasis progression was monitored by BLI and X-ray imaging. Red arrows

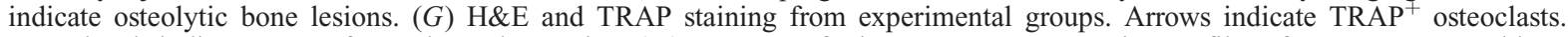
Arrowheads indicate areas of overt bone destruction. $(H)$ Heatmap of microarray gene expression profiles of MC3T3-E1 osteoblasts that were sorted from cocultures of each experimental group (with coculture with control of Jagged1-overexpressing cancer cells, with or without $\alpha$-secretase inhibitor [GSI] treatment). (I) A list of genes selected from $H$ with expression levels greater than threefold in osteoblasts cocultured with Jagged 1-overexpressing tumor cells versus with control tumor cells. $(J)$ Control or Jagged1 overexpressing tumor cells were cocultured with Raw264.7 preosteoclast cells. Dimethylsulfoxide (DMSO) or GSI were immediately added into coculture. Representative TRAP staining images were shown from the experiment. $(K)$ Control or Jagged1-overexpressing SCP28 cells were inoculated into nude mice by intracardiac injection and then treated with either control or GSI (MRK-003). BLI, X-ray, $\mathrm{H} \& \mathrm{E}$, and TRAP staining images of bone lesions from representative mice in each experimental group were displayed on day 42 . TGF- $\beta$, transforming growth factor $\beta$; R-Luc $\left(\mathrm{R}_{\mathrm{LUC}}\right)$, Renilla luciferase; F-Luc $\left(\mathrm{F}_{\mathrm{LUC}}\right)$, firefly luciferase; $\mathrm{KD}$, knockdown; BLI, bioluminescence; H\&E, hematoxylin and eosin; TRAP, tartrate-resistant acid phosphatase. (Adapted, with permission, from Korpal et al. 2009; Sethi et al. 2011.) 


\section{OSTEOCLAST microRNAS AS BIOMARKER AND REGULATOR OF BONE METASTASIS}

Our previous studies mostly focused on protein-coding genes in bone metastasis. However, with better understanding of the human and mouse genomes, microRNAs, long noncoding RNAs, and other regulatory RNAs have been recognized for their important functional roles in many biological processes, including cancer progression and metastasis. Different from regular protein-coding mRNAs, microRNAs are very stable in the circulation, making microRNA-based biomarker and therapeutic development a promising direction in metastasis. Based on the central importance of osteoclasts in osteolytic bone metastasis, we started by investigating how tumor-derived factors affect miRNA expression during osteoclast maturation. We first used conditional media (CM) from highly bone-metastatic cancer cell lines $4 \mathrm{~T} 1.2$ and TSuPr1-B2 to treat RAW264.7 preosteoclast cells at the limited level of RANKL at a concentration that is not sufficient to induce full osteoclastogenesis. CM treatment significantly promoted osteoclast differentiation when combined with a low level of RANKL (Ell et al. 2013). In contrast, $\mathrm{CM}$ from weakly bone-metastatic counterpart cell lines (4T1 and TSU-Pr1 cells) had no effect on osteoclast differentiation. Similar results were also observed in tumor-preosteoclast coculture assay. We performed miRNA microarray analysis of Raw264.7 cells treated with $\mathrm{CM}$ from either highly or weakly bone-metastatic cells. Among the detected microRNAs, 42 miRNAs were up-regulated and 45 miRNAs were down-regulated with a $>2.2$-fold change across treatment groups. Eventually, five down-regulated miRNAs with multiple osteoclast regulatory gene targets, including miR-33a-5p, miR133a, miR-141-3p, miR-190, and miR-219-5p (hereafter miR-33a, miR-141, and miR-219), were confirmed to block osteoclastogenesis when transfected into preosteoclasts, and were further validated in the in vitro bone resorption assays. The strongest repressing effect was seen in miR-133a, miR-141, and miR-219-transfected cells, whereas the other two miRNAs showed mild inhibition. Nevertheless, we proceeded to evaluate all five miRNAs' effect on bone development and bone metastasis in vivo. First, we injected pre-miRNA via tail-vein injection to Balb/c mice weekly for $4 \mathrm{wk}$. The bone density of these mice was evaluated by weekly X-ray imaging and by micro-CT at $4 \mathrm{wk}$. These examinations revealed increased trabecular bone density, bone volume, and thickness in hind limb bones in miRNA treated mice, especially those injected with miR-141, mir-190, or miR219 (Fig. 5A). These changes were associated with decreased TRAP-positive osteoclasts in the bone, whereas no obvious difference in osteoblast activity was observed. The same five miRNAs were subsequently analyzed for their possible effect on reducing bone metastasis. Treatment with MiR-141 or MiR-219 significantly decreased bone-metastasis burden in hind limbs and drastic reduction of TRAP-positive osteoclast number in the bone (Fig. 5B-E). Importantly, among miRNAs up-regulated during osteoclastogenesis, miR-16 and miR-378 can be used as serum biomarkers to detect bone metastasis in breast cancer patients. Our result supports the further development of miRNAs as biomarkers and therapeutic agents of bone metastasis.

\section{BONE-METASTATIC NICHES-LOOKING BEYOND OSTEOCLASTOGENESIS}

The new candidate therapeutic targets of bone metastasis described above were mainly derived from a better understanding of the molecular mechanisms for the "vicious cycle" of bone metastasis. Bone is a nurturing yet extremely complicated microenvironment for metastasis progression and contains many other stromal cells. In normal physiological conditions, bone is not only the structural framework to support the human body but also harbors most of the hematopoietic stem cells (HSCs) for the renewal and lineage differentiation to constitute the whole hematopoietic system (Morrison and Scadden 2014). Studies suggest there are multiple bone stromal cell types occupying specific regions, termed "niches," to support HSC formation. Accumulating evidence in recent research indicated that there are two major nichesthe perivascular niche and the osteoblast niche (Calvi et al. 2003; Zhang et al. 2003). The osteoblast niche is localized at the inner surface of the bone cavity and potentially at the trabecular bone surfaces. Quiescent HSCs are initially proposed to reside in the osteoblast niche. However, recent studies suggest these HSCs are localized to the perivascular niche, which is composed of an endothelial cell, a mesenchymal stromal cell (MSC), and some neuronal cells. Many stem cell factors, such as CXCL12 and stem cell factor (SCF), were produced by a combination of these perivascular niche cells and to some lower extent by osteoblast niche cells (Ding et al. 2012; Greenbaum et al. 2013; Morrison and Scadden 2014). Notably, it has been reported that CXCL12 is a bone-derived chemokine that promotes the bone homing of metastatic tumor cells expressing its receptors with CXCR4 and CXCR7. (Muller et al. 2001; Wang et al. 2006).

As the seeds for distant metastasis, circulating and disseminated tumor cells face a hostile environment during the metastatic cascade, such as the sheer stress of the blood flow and the attacks from immune cells (Gay and Felding-Habermann 2011). When tumor cells eventually arrive at the bone microenvironment, how these cells interact with bone niches is an essential question for understanding the microenvironment for bone metastasis, especially at the early stages of bone metastasis and in therapy resistance. It seems that the interaction with either the perivascular niche or the osteoblast niche could be important in different models (Fig. 6). In a prostate-metastasis model, bone-metastatic tumor cells interact with osteoblast niche cells and prevent the engraftment of the introduced HSC cells (Shiozawa et al. 2011). Interestingly, when HSCs were inoculated into the bone of lethally irradiated mice to rescue the mice from mortality, its protection effect could be reversed by simultaneously inoculating with bone-metastatic cells. Direct imaging 


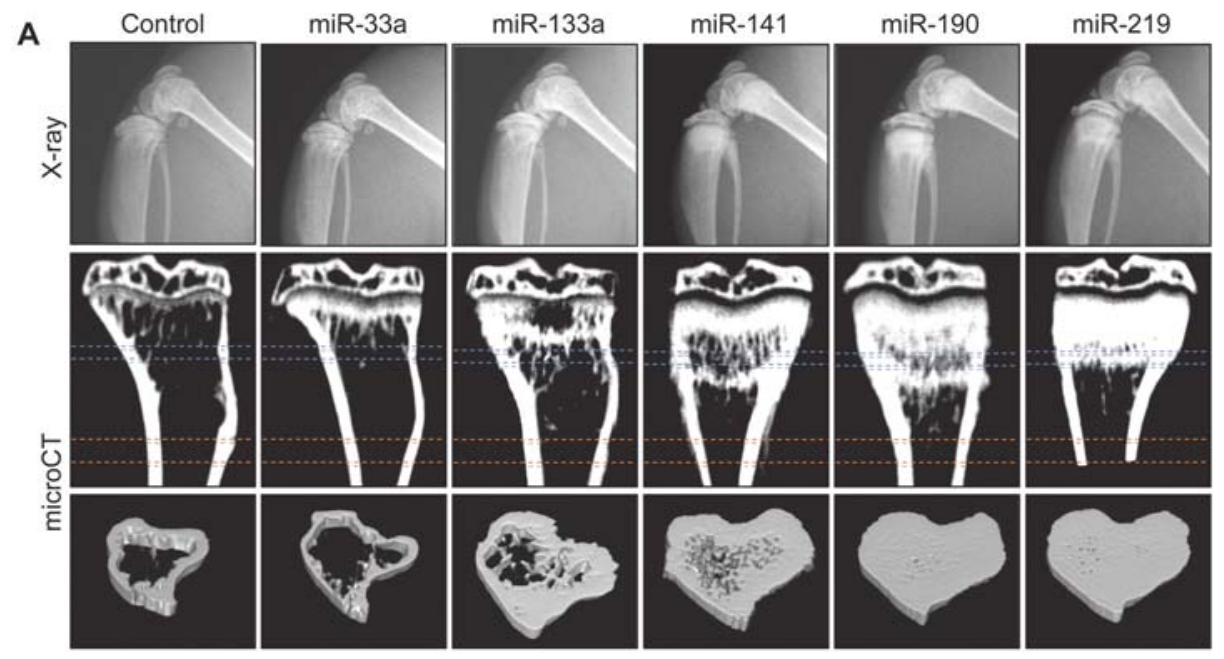

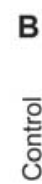
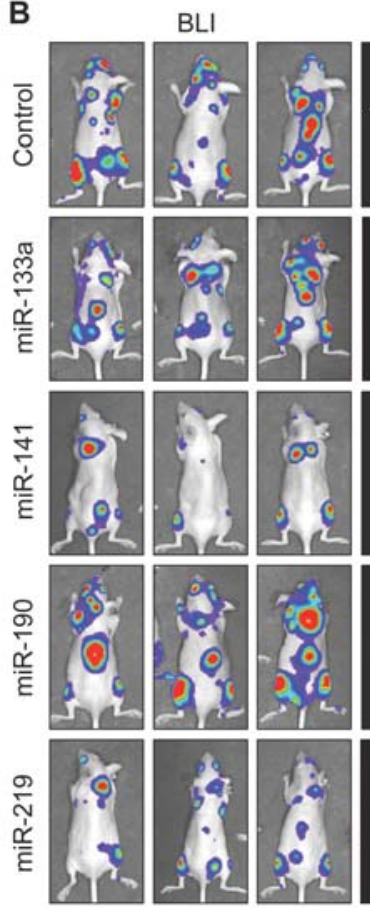
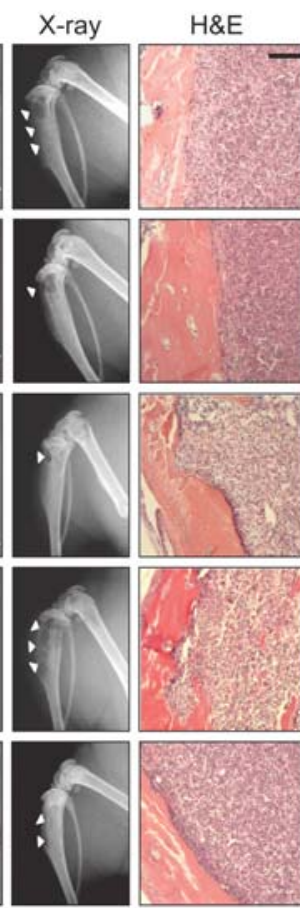

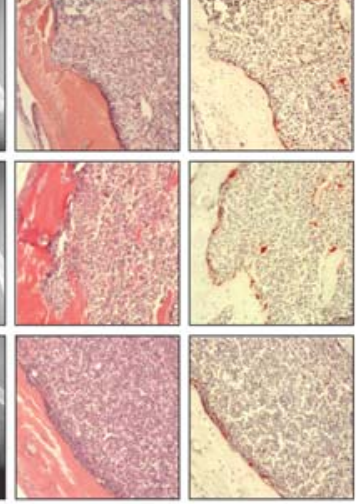

C
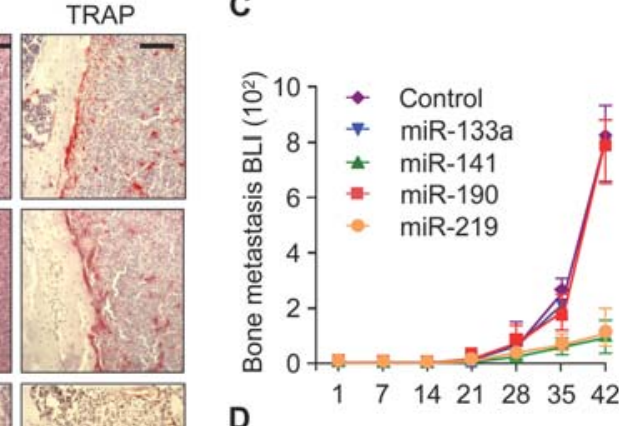

D
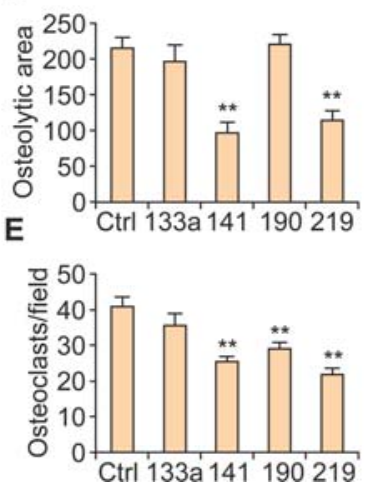

Figure 5. MicroRNAs as therapeutic agents for blocking bone-metastasis progression. $(A)$ Representative X-ray and micro-computed tomography $(\mu \mathrm{CT})$ images of bones from mice treated with indicated miRNAs or a nontargeting control miRNA. $(B)$ SCP28 cells were injected into nude mice to generate bone metastasis and subsequently treated with indicated miRNAs. BLI, X-ray, H\&E, and TRAP images from each group were presented. White arrows indicate osteolytic areas in the X-ray images. $(C)$ Normalized bioluminescence (BLI) signals from mice in $A$. $(D)$ Quantification of osteolytic lesion areas. ${ }^{* *}, P<0.01$. (E) Quantification of TRAP-positive osteoclasts from TRAP staining experiment in $A .{ }^{* *}, P<0.01$. H\&E, hematoxylin and eosin; TRAP, tartrate-resistant acid phosphatase. (Adapted, with permission, from Ell et al. 2013.)

of these cells in the bone niche suggests that they prefer to lodge to similar regions of the bone, most likely to the osteoblast cells. In breast cancer, disseminated tumor cells engage the osteogenic niches in bone through heterotypic adherin junctions (E-cadherin from tumor cells and $\mathrm{N}$-cadherin from osteoblast niche cells). The activation of the mTOR pathway in tumor cells by this interaction fuels the initial metastatic expansion (Wang et al. 2015). When N-E heterotypic cadherin junctions were disengaged by administration of E-cadherin neutralizing antibody, bone-metastasis seeding and colonization were significantly prohibited.

Bone-metastatic tumor cells also engage perivascular niche cells for multiple functions. For example, leukemia cells in the bone marrow localize to and disrupt the normal HSC perivascular niche. In mice bearing leukemia cells, the number of $\mathrm{CD} 34^{+}$cells drastically decreased over time and were not able to enter the peripheral circulation upon stimulation (Colmone et al. 2008). In the cancer dormancy model, dormant disseminated tumor 


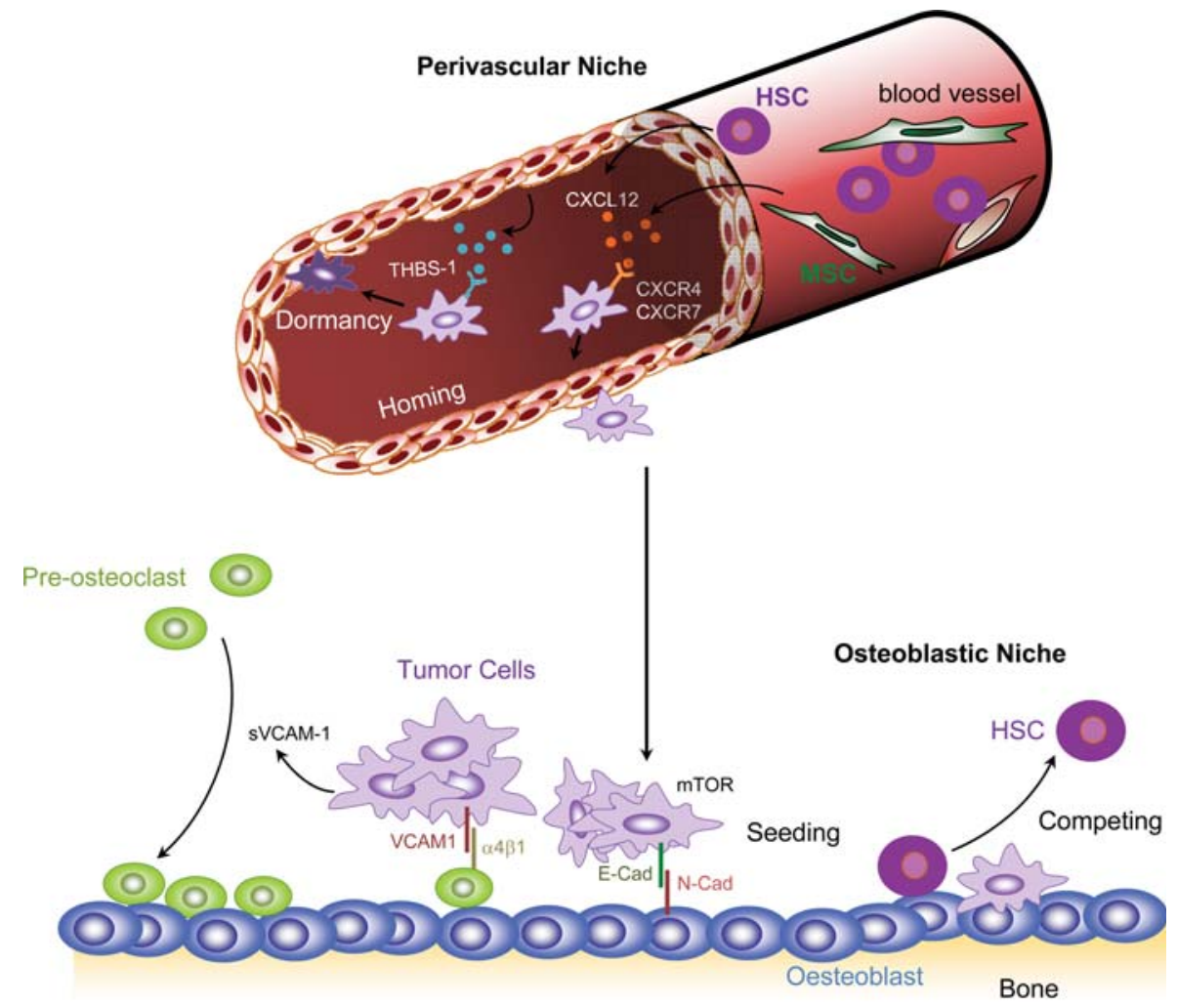

Figure 6. Various bone niches and their role in bone metastasis. In the perivascular niche, CXCL12 and CXCR4/CXCR7 interaction is critical for tumor cell homing to the bone. Tumor dormancy is mediated by endothelial-derived thrombospondin-1. The osteoblastic niche is critical for initial tumor seeding and micrometastatic growth in prostate and breast cancer. The N/E-cadherin junction between tumor and osteoblasts promotes micrometastatic tumor growth through activating the AKT-mTOR pathway. Tumor-derived VCAM1 attracts preosteoclasts and promotes their differentiation to facilitate osteolytic outgrowth. HSC, hematopoietic stem cell; VCAM1, vascular cell adhesion molecule 1; mTOR, mammalian target of rapamycin.

cells (DTCs) reside on the vasculature of metastatic sites, including the bone (Ghajar et al. 2013). The interaction of tumor cells with microvascular niche cells, specifically mediated by endothelial-derived thrombospondin-1, leads to tumor cell quiescence. However, this repressive signal is lost upon the sprouting of neovasculature and the switch to a growth-permissive microenvironment. Our recent analysis of metastatic dormancy and activation in bone also revealed an important role of VCAM-1 in this process. Tumor-derived VCAM-1 attracts monocytic osteoclast progenitor cells to promote osteoclast activity so that the tumor cells can outgrow from indolent micrometastasis to form overt metastasis (Lu et al. 2011). Despite these new insights regarding different niche environments in bone metastasis, the temporospatial dynamics of tumor cell occupancy and utilization of these niches during different stages of metastatic progression require further investigations.

Systematic cancer therapy, like hormone therapy, targeted therapy, and chemotherapy, as well as localized radiation therapy, could reduce bone-metastasis incidence, slow down metastatic growth, and most importantly alleviate some of the symptoms caused by metastasis. However, these treatments also have other unavoidable effects on bone stromal components. Whether the changes in the bone niche cells have a negative or positive effect on metastasis progression is an important yet poorly explored question. A few recent studies showed that there are fundamental changes in bone niche cells during chemotherapy of hematopoietic malignancies, which promote chemoresistance. Treatment of bone engrafted acute lymphoblastic leukemia (ALL) with cytarabine or daunorubicin for a few days induced a striking bone niche switch (Duan et al. 2014). At the early stage of the treatment, ALL cells were mainly localized at the normal bone niches of vascular type. However, $12 \mathrm{~d}$ after treatment, most residual cells were present in locations full of mesenchymal cell types. Molecular characterization of these cells revealed they are $\mathrm{Nestin}^{+} \mathrm{NG} 2^{-}$mesenchymal cells, which express high level of GDF15 to activate the TGF- $\beta$ pathway in the leukemia cells and protect the cells from chemotherapy-induced apoptosis. Other studies also suggest that systematic therapy could induce chemokines and growth factors from bone stromal cells, including CXCL-12, CCL2, VEGF, and hepatocyte growth factor (HGF), to inhibit apoptosis (Jankowski et al. 2003; Park et al. 2012). A deeper understanding is needed to reveal the complete tumor-bone niche interaction and modifications during every major step of metastasis and in the development of therapy resistance. This knowledge will serve as the foundation to improve targeted therapies for bone metastasis. 


\section{CONCLUSION}

With the development of bone-metastatic cell lines, in vivo BLI imaging, and $\mu \mathrm{CT}$ imaging of the mice, we are able to study bone metastasis in preclinical mouse models qualitatively and quantitatively. Genomic profiling of in vivo selected bone-metastatic variants combined with functional analysis in mouse models and clinical prognosis studies have led to the identification and validation of multiple novel bone-metastasis genes with diverse functional mechanisms. MMP1 and ADMATS1 proteolytically release membrane-bound EGF ligands to promote osteoclast differentiation. Tumor-derived Jagged1, which is induced by bone-derived TGF- $\beta$, promotes osteoclastogenesis and survival cytokine production from osteoblast cells. VCAM-1 expression in disseminated tumor cells and bone micrometastases can be activated in inflammatory cytokines and functions to recruit and activate preosteoclasts to promote the aggressive outgrowth of bone metastasis. miRNAs involved in regulating osteoclast maturation can also be used as biomarkers and therapeutic agents of bone metastasis. These basic understandings of bone metastasis have revealed multiple new druggable targets for reducing or blocking bone metastasis, including TGF- $\beta$, Jagged $1-$ Notch, and VCAM-1.

Recent studies also revealed two critical niches in HSC development - the perivascular niche and the osteoblastic niche. We have started to appreciate the importance of these two niches in attracting tumor cells to the bone, initializing bone seeding, maintaining and breaking the dormancy, and promoting bone-metastasis outgrowth and therapy resistance. Further understanding of the molecular mechanisms in each of these steps will undoubtedly provide additional new targets for future drug development.

\section{ACKNOWLEDGMENTS}

We thank members of the Kang Laboratory for a critical reading of the manuscript. Research in our laboratory is supported by grants from the National Institutes of Health, Department of Defense, Susan G. Komen Foundation, and the Brewster Foundation. H.Z. was supported by a Komen Postdoctoral Fellowship (KG111164).

\section{REFERENCES}

Baron R, Ferrari S, Russell RG. 2011. Denosumab and bisphosphonates: Different mechanisms of action and effects. Bone 48: $677-692$.

Cailleau R, Olive M, Cruciger QV. 1978. Long-term human breast carcinoma cell lines of metastatic origin: Preliminary characterization. In vitro 14: $911-915$.

Calvi LM, Adams GB, Weibrecht KW, Weber JM, Olson DP, Knight MC, Martin RP, Schipani E, Divieti P, Bringhurst FR, et al. 2003. Osteoblastic cells regulate the haematopoietic stem cell niche. Nature 425: 841-846.

Colmone A, Amorim M, Pontier AL, Wang S, Jablonski E, Sipkins DA. 2008. Leukemic cells create bone marrow niches that disrupt the behavior of normal hematopoietic progenitor cells. Science 322: 1861-1865.
Ding L, Saunders TL, Enikolopov G, Morrison SJ. 2012. Endothelial and perivascular cells maintain haematopoietic stem cells. Nature 481: 457-462.

Duan CW, Shi J, Chen J, Wang B, Yu YH, Qin X, Zhou XC, Cai YJ, Li ZQ, Zhang F, et al. 2014. Leukemia propagating cells rebuild an evolving niche in response to therapy. Cancer Cell 25: $778-793$.

Ell B, Kang Y. 2012. SnapShot: Bone metastasis. Cell 151: 690-690.e691.

Ell B, Mercatali L, Ibrahim T, Campbell N, Schwarzenbach H, Pantel K, Amadori D, Kang Y. 2013. Tumor-induced osteoclast miRNA changes as regulators and biomarkers of osteolytic bone metastasis. Cancer Cell 24: 542-556.

Engin F, Yao Z, Yang T, Zhou G, Bertin T, Jiang MM, Chen Y, Wang L, Zheng H, Sutton RE, et al. 2008. Dimorphic effects of Notch signaling in bone homeostasis. Nat Med 14: 299305.

Gay LJ, Felding-Habermann B. 2011. Contribution of platelets to tumour metastasis. Nat Rev Cancer 11: 123-134.

Ghajar CM, Peinado H, Mori H, Matei IR, Evason KJ, Brazier H, Almeida D, Koller A, Hajjar KA, Stainier DY, et al. 2013. The perivascular niche regulates breast tumour dormancy. Nat Cell Biol 15: 807-817.

Greenbaum A, Hsu YM, Day RB, Schuettpelz LG, Christopher MJ, Borgerding JN, Nagasawa T, Link DC. 2013. CXCL12 in early mesenchymal progenitors is required for haematopoietic stem-cell maintenance. Nature 495: 227-230.

Hilton MJ, Tu X, Wu X, Bai S, Zhao H, Kobayashi T, Kronenberg HM, Teitelbaum SL, Ross FP, Kopan R, et al. 2008. Notch signaling maintains bone marrow mesenchymal progenitors by suppressing osteoblast differentiation. Nat Med 14: $306-314$.

Jankowski K, Kucia M, Wysoczynski M, Reca R, Zhao D, Trzyna E, Trent J, Peiper S, Zembala M, Ratajczak J, et al. 2003. Both hepatocyte growth factor (HGF) and stromal-derived factor-1 regulate the metastatic behavior of human rhabdomyosarcoma cells, but only HGF enhances their resistance to radiochemotherapy. Cancer Res 63: 79267935.

Kang Y. 2016. Dissecting tumor-stromal interactions in breast cancer bone metastasis. Endocrinol Metab 31: 206-212.

Kang Y, Siegel PM, Shu W, Drobnjak M, Kakonen SM, CordonCardo C, Guise TA, Massague J. 2003. A multigenic program mediating breast cancer metastasis to bone. Cancer Cell 3: $537-549$.

Korpal M, Yan J, Lu X, Xu S, Lerit DA, Kang Y. 2009. Imaging transforming growth factor- $\beta$ signaling dynamics and therapeutic response in breast cancer bone metastasis. Nat Med 15: 960-966.

Lu X, Wang Q, Hu G, Van Poznak C, Fleisher M, Reiss M, Massague J, Kang Y. 2009. ADAMTS1 and MMP1 proteolytically engage EGF-like ligands in an osteolytic signaling cascade for bone metastasis. Genes Dev 23: 1882-1894.

Lu X, Mu E, Wei Y, Riethdorf S, Yang Q, Yuan M, Yan J, Hua Y, Tiede BJ, Lu X, et al. 2011. VCAM-1 promotes osteolytic expansion of indolent bone micrometastasis of breast cancer by engaging $\alpha 4 \beta 1$-positive osteoclast progenitors. Cancer Cell 20: 701-714.

Melton LJ III, Kyle RA, Achenbach SJ, Oberg AL, Rajkumar SV. 2005. Fracture risk with multiple myeloma: A populationbased study. J Bone Miner Res 20: 487-493.

Morrison SJ, Scadden DT. 2014. The bone marrow niche for haematopoietic stem cells. Nature 505: 327-334.

Muller A, Homey B, Soto H, Ge N, Catron D, Buchanan ME, McClanahan T, Murphy E, Yuan W, Wagner SN, et al. 2001. Involvement of chemokine receptors in breast cancer metastasis. Nature 410: 50-56.

Mundy GR. 2002. Metastasis to bone: Causes, consequences and therapeutic opportunities. Nat Rev Cancer 2: 584-593.

Park SI, Liao J, Berry JE, Li X, Koh AJ, Michalski ME, Eber MR, Soki FN, Sadler D, Sud S, et al. 2012. Cyclophosphamide creates a receptive microenvironment for prostate cancer skeletal metastasis. Cancer Res 72: 2522-2532. 
Ren G, Esposito M, Kang Y. 2015. Bone metastasis and the metastatic niche. J Mol Med 93: 1203-1212.

Sethi N, Kang Y. 2011. Unravelling the complexity of metastasismolecular understanding and targeted therapies. Nat Rev Cancer 11: $735-748$.

Sethi N, Dai X, Winter CG, Kang Y. 2011. Tumor-derived JAGGED1 promotes osteolytic bone metastasis of breast cancer by engaging notch signaling in bone cells. Cancer Cell 19: $192-205$.

Shiozawa Y, Pedersen EA, Havens AM, Jung Y, Mishra A, Joseph J, Kim JK, Patel LR, Ying C, Ziegler AM, et al. 2011. Human prostate cancer metastases target the hematopoietic stem cell niche to establish footholds in mouse bone marrow. J Clin Invest 121: 1298-1312.

Wan L, Pantel K, Kang Y. 2013. Tumor metastasis: Moving new biological insights into the clinic. Nat Med 19: 14501464.
Wang J, Loberg R, Taichman RS. 2006. The pivotal role of CXCL12 (SDF-1)/CXCR4 axis in bone metastasis. Cancer Metastasis Rev 25: 573-587.

Wang H, Yu C, Gao X, Welte T, Muscarella AM, Tian L, Zhao H, Zhao Z, Du S, Tao J, et al. 2015. The osteogenic niche promotes early-stage bone colonization of disseminated breast cancer cells. Cancer Cell 27: 193-210.

Weilbaecher KN, Guise TA, McCauley LK. 2011. Cancer to bone: A fatal attraction. Nat Rev Cancer 11: 411-425.

Wu H, Bui MM, Leston DG, Shao H, Sokol L, Sotomayor EM, Zhang L. 2014. Clinical characteristics and prognostic factors of bone lymphomas: Focus on the clinical significance of multifocal bone involvement by primary bone large B-cell lymphomas. BMC Cancer 14: 900.

Zhang J, Niu C, Ye L, Huang H, He X, Tong WG, Ross J, Haug J, Johnson T, Feng JQ, et al. 2003. Identification of the haematopoietic stem cell niche and control of the niche size. Nature 425: 836-841. 


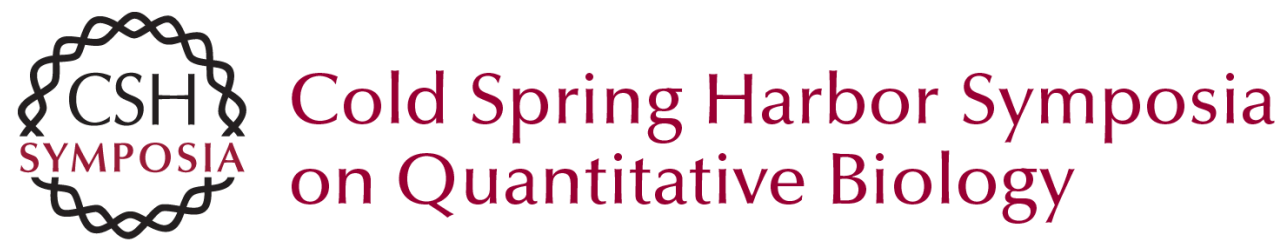

\section{Tumor-Stroma Interactions in Bone Metastasis: Molecular Mechanisms and Therapeutic Implications}

Hanqiu Zheng, Wenyang Li and Yibin Kang

Cold Spring Harb Symp Quant Biol 2016 81: 151-161 originally published online April 5, 2017 Access the most recent version at doi:10.1101/sqb.2016.81.030775

References This article cites 34 articles, 4 of which can be accessed free at: http://symposium.cshlp.org/content/81/151.full.html\#ref-list-1

Creative This article is distributed under the terms of the

Commons http://creativecommons.org/licenses/by-nc/4.0/, which permits reuse and License redistribution, except for commercial purposes, provided that the original author and source are credited.

Email Alerting Receive free email alerts when new articles cite this article - sign up in Service the box at the top right corner of the article or click here. 